\title{
Revisión sistemática de los biomarcadores y su asociación con el síndrome metabólico en México
}

\author{
ANa Ligia GutiéRREZ-Solís* \\ Departamento de Investigación, Hospital Regional de Alta Especialidad de la Península de Yucatán (HRAEPY), Mérida, Yuc., México
}

\begin{abstract}
RESUMEN
Introducción: El síndrome metabólico (SM) se diagnostica cuando se presentan al menos tres de las siguientes cinco anormalidades: obesidad abdominal, resistencia a la insulina (RI), hipertensión arterial, hiperglicemia y dislipidemia. EI SM aumenta el riesgo de desarrollar enfermedades cardiovasculares (ECV) y diabetes mellitus tipo 2 (DM2); además, es un factor de riesgo para otras enfermedades de alta mortalidad. Estas anormalidades pueden ser detectadas y monitoreadas por medio de biomarcadores séricos. Esta revisión congrega un grupo de marcadores séricos que han mostrado una asociación y utilidad para la detección temprana del SM. Métodos: Se realizó una revisión sistemática en PubMed y Google Scholar. Los biomarcadores incluidos en la búsqueda fueron: leptina, resistina (RSTN), adiponectina, ácido úrico, inhibidor del activador del plasminógeno 1 (PAI-1), interleucina (IL) 6, proteína C reactiva (PCR), IL-10, factor de necrosis tumoral $\alpha$ (TNF- $\alpha$ ), paraoxonasa 1 (PON-1) y LDL oxidada (LDLox). Resultados: Los niveles de leptina, RSTN, PAI-1, ácido úrico, IL-6, PCR, TNF- $\alpha$ y LDLox se encontraron elevados en estudios que examinaron a individuos con SM. Los niveles de adiponectina, ghrelina, IL-10 y PON-1 se encontraron disminuidos en individuos con SM. Conclusión: Todos los biomarcadores presentaron una asociación con el SM. Sin embargo, se necesita más investigación para determinar su eficacia para el diagnóstico del SM.
\end{abstract}

Palabras clave: Síndrome metabólico. Biomarcadores. Revisión de la literatura.

\begin{abstract}
Introduction: Metabolic Syndrome (MetS) is diagnosed when 3 out of 5 of the following abnormalities are presented, including abdominal obesity, insulin resistance, hypertension, hyperglycemia, and dyslipidemia. MetS increases the risk of developing cardiovascular disease and diabetes mellitus 2. Besides, it is also a risk factor for other diseases with high mortality. Abnormalities can be detected and monitored via serum biomarkers. This review collected some biomarkers that have been shown to have an association and utility for the early detection of MS. Methods: We performed a systematic review in PubMed and Google Scholar. The biomarkers included in the search were leptin, resistin, adiponectin, uric acid, PAI-1, IL-6, PCR, IL-10, TNF $\alpha$, PON-1 and oxLDL. Results: Levels of leptin, resistin, PAI-1, uric acid, IL-6, CRP, TNF- $\alpha$ and oxLDL are elevated in subjects with SM. Levels of adiponectin, ghrelin, IL-10 and
\end{abstract}

\section{Correspondence:}

*Ana Ligia Gutiérrez-Solís

Hospital Regional de Alta Especialidad de la Península de Yucatán

Calle 7, 433 por 20 y 22, Fracc. Altabrisa

C.P. 97130 , Mérida, Yuc., México

E-mail: ganaligia@gmail.com

Received for publication: 12-09-2017

Accepted for publication: 16-03-2018

DOI:10.24875/HMCM.18000091 
PON-1 are found decreased in subjects with SM. Conclusion: The biomarkers showed an association with MetS. However, more research is needed to determine the efficacy of biomarkers for the diagnosis of MetS. (Hosp Med Clin Manag. 2018;11:27-35)

Corresponding author: Ana Ligia Gutiérrez-Solís, ganaligia@gmail.com

Key words: Metabolic syndrome. Biomarkers. Literature review.

\section{INTRODUCCIÓN}

El SM se caracteriza por la congregación de anormalidades que incrementan la probabilidad de un individuo de desarrollar enfermedades de alta mortalidad. Se ha encontrado que los individuos con SM aumentan aproximadamente al doble la probabilidad de padecer ECV ${ }^{1}$. Además, la obesidad abdominal y la RI han sido señaladas como algunos de los factores causantes del $\mathrm{SM}^{2}$. Sin embargo, la patogénesis del SM y sus componentes aún no se comprende en su totalidad. Diferentes organizaciones han definido y descrito los componentes del SM (Tabla 1); los criterios evaluados son ${ }^{3}$ :

- Obesidad (circunferencia de la cintura o índice de masa corporal [IMC]).

- Niveles de triglicéridos.

- Niveles de colesterol unido a lipoproteínas de alta densidad (C-HDL).

- Hipertensión arterial.

- Hiperglucemia.

Aunque diversas organizaciones mundiales han definido el SM, entre las definiciones más utilizadas se encuentran la del Panel de Tratamiento para Adultos III del Programa Nacional para la Educación sobre el Colesterol (National Cholesterol Education Program's Adult Treatment Panel III [NCEP-ATP III]), la de la Federación Internacional de Diabetes (International Diabetes Federation [IDF]) y la de la Organización Mundial de la Salud $(\mathrm{OMS})^{3}$. Dependiendo de la definición y el criterio utilizados se pueden encontrar diferencias en las prevalencias en las poblaciones a nivel mundial e incluso a nivel regional. Los estudios han demostrado que los individuos con SM incrementan el riesgo de desarrollar DM2 y ECV4. Además, estos individuos también son más susceptibles a desarrollar otras condiciones como síndrome de ovario poliquístico, apnea de sueño, hígado graso y algunos tipos de cáncer, como el pancreático, el colorrectal y el de próstata ${ }^{5-7}$.

En México, los estudios epidemiológicos muestran una prevalencia del SM de entre el 20 y el 50\%, dependiendo de la definición utilizada, la edad y el área poblacional analizada 8 . Asimismo, los resultados en adultos mayores de 20 años del ENSANUT 2016 muestran prevalencias altas en algunos de los componentes del SM; por ejemplo, se encontró una prevalencia del 25.5\% entre la población mexicana con hipertensión arterial y la prevalencia combinada de sobrepeso y obesidad pasó del $71.2 \%$ en 2012 al $72.5 \%$ en $2016^{9}$. Dadas las altas prevalencias de estas enfermedades en México, es necesario encontrar estrategias para la reducción de las complicaciones que conlleva el SM. La detección temprana es primordial para este proceso; sin embargo, frecuentemente el diagnóstico sólo es posible una vez que las complicaciones ya han comenzado.

En la actualidad, la medicina utiliza la ayuda de ciertos biomarcadores para el diagnóstico y manejo de diversas enfermedades, especialmente cuando hay ausencia de signos clínicos. También se ha descrito la utilidad de los biomarcadores para la identificación de individuos susceptibles a padecer ciertas enfermedades dentro de una población ${ }^{10}$.

Entre las diversas sustancias que pueden utilizarse como biomarcadores, algunos estudios han mencionado la utilidad de los adipocitos, ya que producen sustancias bioactivas conocidas como adipocitocinas o adipocinas ${ }^{11}$. La acumulación de adipocitos conlleva una producción desregulada de adipocinas, lo que contribuye al desarrollo de la obesidad ${ }^{12}$. Actualmente, el mecanismo por el cual la acumulación adiposa provoca esta desregulación no está totalmente claro, pero los estudios sugieren que el estrés oxidativo sistémico desempeña un papel importante en el desarrollo de la obesidad $^{13}$. El estrés oxidativo se manifiesta por un 
Tabla 1. Criterio para el diagnóstico del SM

\begin{tabular}{|c|c|c|c|c|}
\hline & IDF (obesidad $+\geq 2$ ) & AHA/NHLBI $(\geq 3)$ & ATP III $(\geq 3)$ & OMS (RI o DM2 + $\geq 2$ ) \\
\hline Obesidad & $\begin{array}{l}\mathrm{IMC}>30 \mathrm{~kg} / \mathrm{m}^{2} \text { o perímetro } \\
\text { de la cintura: con especificidad } \\
\text { respecto a los distintos grupos } \\
\text { étnicos }\end{array}$ & $\begin{array}{l}\text { Perímetro de la } \\
\text { cintura: hombres }>102 \mathrm{~cm} \text {, } \\
\text { mujeres }>88 \mathrm{~cm}\end{array}$ & $\begin{array}{l}\text { Perímetro de la } \\
\text { cintura: hombres }>102 \mathrm{~cm} \text {, } \\
\text { mujeres }>88 \mathrm{~cm}\end{array}$ & $\begin{array}{l}\text { Perímetro de la cintura/ } \\
\text { cadera: hombres }>0.90 \mathrm{~cm} \text {, } \\
\text { mujeres }>0.85 \mathrm{~cm} 0 \\
\mathrm{IMC}>30 \mathrm{~kg} / \mathrm{m}^{2}\end{array}$ \\
\hline $\begin{array}{l}\text { Niveles altos de } \\
\text { triglicéridos }\end{array}$ & $\begin{array}{l}\geq 150 \mathrm{mg} / \mathrm{dl} \text { o tratamiento } \\
\text { específico para la anormalidad }\end{array}$ & $\begin{array}{l}\geq 150 \mathrm{mg} / \mathrm{dl} \text { o tratamiento } \\
\text { específico para la anormalidad }\end{array}$ & $\begin{array}{l}\geq 150 \mathrm{mg} / \mathrm{dl} \text { o tratamiento } \\
\text { específico para la anormalidad }\end{array}$ & $\geq 150 \mathrm{mg} / \mathrm{dl}$ \\
\hline Niveles bajos de & Hombres $<40$ mg/dl & Hombres $<40 \mathrm{mg} / \mathrm{dl}$ & Hombres $<40$ mg/dl & Hombres $<35$ mg/dl \\
\hline C-HDL & $\begin{array}{l}\text { Mujeres }<50 \mathrm{mg} / \mathrm{dl} \\
\text { o tratamiento específico } \\
\text { para la anormalidad }\end{array}$ & $\begin{array}{l}\text { Mujeres }<50 \mathrm{mg} / \mathrm{dl} \\
\text { o tratamiento específico } \\
\text { para la anormalidad }\end{array}$ & $\begin{array}{l}\text { Mujeres }<50 \mathrm{mg} / \mathrm{dl} \\
\text { o tratamiento específico } \\
\text { para la anormalidad }\end{array}$ & Mujeres < 39 mg/dl \\
\hline $\begin{array}{l}\text { Hipertensión } \\
\text { arterial }\end{array}$ & $\begin{array}{l}\geq 130 / \geq 85 \mathrm{mmHg} \\
0 \text { tratamiento farmacológico } \\
\text { para la condición }\end{array}$ & $\begin{array}{l}\geq 130 / \geq 85 \mathrm{mmHg} \\
0 \text { tratamiento farmacológico } \\
\text { para la condición }\end{array}$ & $\begin{array}{l}\geq 130 / \geq 85 \mathrm{mmHg} \\
0 \text { tratamiento farmacológico } \\
\text { para la condición }\end{array}$ & $\geq 140 / \geq 90 \mathrm{mmHg}$ \\
\hline Hiperglucemia & $\begin{array}{l}\text { Glucemia en ayunas } \\
\geq 100 \mathrm{mg} / \mathrm{dl} \text { o bien DM2 } \\
\text { diagnosticada previamente }\end{array}$ & $\begin{array}{l}\text { Glucemia en ayunas } \\
\geq 100 \mathrm{mg} / \mathrm{dl} \text { o bien DM2 } \\
\text { diagnosticada previamente }\end{array}$ & $\begin{array}{l}\text { Glucemia en ayunas } \\
\geq 100 \mathrm{mg} / \mathrm{dl} 0 \text { bien DM2 } \\
\text { diagnosticada previamente }\end{array}$ & $\mathrm{RI}$ \\
\hline
\end{tabular}

AHA/NHLBI: American Heart Association and the National Heart Lung and Blood Institute. DM2: diabetes mellitus tipo 2; RI: resistencia a la Insulina.

aumento de especies reactivas de oxígeno (ROS). Las ROS, además de alterar una gran variedad de estructuras celulares, pueden inducir respuestas inflamatorias ${ }^{14}$ que se han relacionado con la $\mathrm{Rl}$ y la diabetes.

La importancia de la utilidad de los biomarcadores radica en que pueden servir como una herramienta para el diagnóstico, monitoreo y tratamiento de individuos con SM y de sus complicaciones. La detección temprana conlleva una intervención oportuna, que resultaría en la disminución de los efectos relacionados con el SM en la población mexicana. Si bien existen numerosos artículos que enumeran diversos biomarcadores, tanto los establecidos hasta el momento como los emergentes, esta revisión conjunta los biomarcadores más investigados en la actualidad y su relación con el SM en la población mundial y en México.

\section{MÉTODOS}

Durante los meses de julio a agosto de 2017 se realizó una revisión de la literatura usando dos buscadores electrónicos, PubMed y Google Scholar. La última búsqueda se realizó el 14 de agosto de 2017. Los términos claves utilizados fueron: biomarcadores, marcadores biológicos, síndrome metabólico, obesidad, leptina, resistina, adiponectina, ghrelina, ácido úrico, inhibidor del activador del plasminógeno 1, interleucina 6, proteína C reactiva, interleucina 10 , factor de necrosis tumoral $\alpha$, paraoxonasa, LDL oxidada y México. Se utilizaron diversas combinaciones de estos términos y las búsquedas se llevaron a cabo en español e inglés. La búsqueda se centró en la población mexicana, pero, debido a la falta o nula información de estos biomarcadores en la población mexicana, en algunas ocasiones se tomó referencia en poblaciones mundiales.

\section{RESULTADOS}

Se encontró en la literatura científica un total de 11 biomarcadores asociados al SM y sus complicaciones. A continuación, se presenta una descripción de estos biomarcadores, su función y su asociación al SM.

\section{Leptina}

La leptina es una hormona que en condiciones fisiológicas normales reduce el apetito, aumenta el gasto energético, facilita la utilización de la glucosa y mejora la sensibilidad a la insulina ${ }^{15}$. Se expresa en niveles proporcionales a la masa adiposa y es producida principalmente por los adipocitos y, en menor medida, por las células del músculo liso vascular, el epitelio gástrico y la glándula mamaria. La isoforma obRb es el receptor funcional y abunda en el hipotálamo, donde cumple la función de aumentar el gasto de energía y reducir el apetito $^{16}$. Los estudios han demostrado que la leptina contribuye a la hipertrofia ventricular izquierda y que puede predecir el infarto de miocardio ${ }^{17}$. También afecta a la estructura vascular promoviendo la hipertensión, 
la angiogénesis y la aterosclerosis ${ }^{18}$. Se han encontrado continuamente niveles elevados de leptina en sujetos con SM; esto ha sido constante entre diversos grupos étnicos y poblaciones. En México, García-Jiménez, et al. realizaron un estudio con 204 trabajadores de la Universidad de Morelos y encontraron una fuerte asociación entre la leptina, el SM y sus componentes, especialmente con la obesidad central y la RI, que son indicadores de trastornos metabólicos como la dislipidemia, la DM2 y la hipertensión arterial ${ }^{19}$. Por todo esto, se considera que la leptina puede servir como biomarcador del SM.

\section{Resistina}

La RSTN es un polipéptido producido por los adipocitos maduros y macrófagos ${ }^{20}$. Se ha vinculado con el estado inflamatorio debido a su predominante producción de monocitos y su correlación con los niveles de IL-621,22. Varios estudios han identificado asociaciones positivas entre los niveles de RSTN y la obesidad, la adipogénesis y la $\mathrm{Rl}^{23}$. En un modelo animal con ratones obesos por una dieta inducida se observó una reducción de los niveles de glucosa y de la acción de la insulina después de la administración del anticuerpo anti-RSTN ${ }^{21}$. Por lo tanto, la RSTN es una hormona que se vincula potencialmente a la obesidad y la diabetes ${ }^{24}$. Sin embargo, el papel que juega en pacientes con SM es un poco contradictorio e inconcluso hasta el momento.

\section{Adiponectina}

La adiponectina, también conocida como Acrp3025, es una proteína plasmática derivada del tejido adiposo y secretada exclusivamente a partir de adipocitos ${ }^{26}$. Tiene un efecto sobre el metabolismo de la glucosa mediante la regulación de la expresión de mARN de las enzimas gluconeogénicas hepáticas ${ }^{27}$. Entre sus funciones están la sensibilización a la insulina, la potenciación de la oxidación de lípidos y la vasodilatación ${ }^{28,29}$. Se han encontrado niveles bajos de adiponectina en individuos con hipertensión arterial y en obesos ${ }^{30}$. Los estudios muestran que la pérdida de peso y una dieta balanceada pueden modificar y elevar los niveles de adiponectina en individuos con obesidad. En un estudio realizado en 700 individuos de entre 6 y 19 años de edad se encontró que la media de adiponectina fue significativamente menor en los pacientes con $\mathrm{SM}^{31}$. Esta tendencia se ha encontrado continuamente en las diferentes poblaciones analizadas. Dado el impacto de la adiponectina en los componentes del SM, podría postularse como un candidato de biomarcador para el diagnóstico del síndrome.

\section{Ghrelina}

La ghrelina es una citocina secretada principalmente por el estómago que estimula el apetito directamente a través de la activación del receptor secretagogo GHR-1a en el hipotálamo e indirectamente mediante el aumento del neuropéptido Y32. Diversos estudios han postulado la ghrelina como una citocina cardioprotectora ${ }^{33}$, ya que se ha asociado a la reducción de la presión arterial ${ }^{34}$. EI SM se asocia con niveles bajos de ghrelina, niveles que pueden disminuir aún más con el número de trastornos presentes, incluyendo la obesidad, la Rl y la hipertensión ${ }^{35-38}$. Sin embargo, la asociación entre los niveles bajos de ghrelina y el SM se explica principalmente por su relación con la obesidad, ya que los sujetos obesos con SM tienen niveles de ghrelina más bajos que los no obesos ${ }^{38}$. En México, hasta el momento no hay estudios que exploren las posibles asociaciones entre la ghrelina y el SM.

\section{Ácido úrico}

El ácido úrico es el producto final del metabolismo de las purinas, formado por el hígado y excretado principalmente por los riñones. A nivel extracelular el ácido úrico tiene capacidades antioxidantes y a nivel intracelular, capacidades proinflamatorias y prooxidantes. El ácido úrico se utiliza como un marcador de riesgo en condiciones patológicas como el hígado isquémico, la aterosclerosis, la diabetes y la insuficiencia cardíaca crónica ${ }^{39}$. Algunos estudios señalan la capacidad del ácido úrico de estimular a los monocitos en la producción de TNF- $\alpha$, creando un estado proinflamatorio ${ }^{40}$. La hiperuricemia es un factor de riesgo para eventos ateroscleróticos como el infarto de miocardio y el accidente cerebrovascular, y para factores de riesgo cardiovascular como la hipertensión arterial y la dislipidemia. Se ha encontrado que los valores altos de ácido úrico desfavorecen el pronóstico de los pacientes con insuficiencia cardíaca ${ }^{41,42}$. Además, varios estudios han asociado niveles altos de ácido úrico en los individuos con SM, niveles que aumentan dependiendo de la presencia del número de componentes del SM. Se estima que las personas con niveles altos de ácido úrico tienen una probabilidad mayor (1.6 veces) de desarrollar $\mathrm{SM}^{42}$. Entre la población mexicana, Jiménez, et al. 
encontraron que la prevalencia del SM se incrementaba sustancialmente con las concentraciones de ácido úrico elevadas ${ }^{43}$.

\section{Inhibidor del activador del plasminógeno 1}

El PAl-1 es el principal de cuatro inhibidores de la serina peptidasa; entre sus funciones se encuentra la remodelación de la matriz extracelular y la fibrinólisis intravascular ${ }^{44}$. Bajo condiciones fisiológicas normales, el PAl-1 es secretado a la circulación o el espacio extracelular por las células endoteliales, los adipocitos, las plaquetas o los hepatocitos, pero bajo condiciones patológicas es inducido por factores proinflamatorios y prooxidantes. Por ejemplo, cuando se encuentran niveles altos de TNF- $\alpha$, factor de crecimiento transformante $\beta$, angiotensina II, glucocorticoides e insulina, esto resulta en la elevación de los niveles de PAI-1. Se ha encontrado que los niveles elevados de PAl-1 están asociados con el riesgo de enfermedad coronaria, ya que contribuye a la formación del trombo ${ }^{45}$. Además, se ha vinculado con la angiogénesis, la metástasis de cáncer, la artritis reumatoide y la enfermedad renal crónica, entre otras ${ }^{46}$

La asociación entre el PAI-1 y el SM se ha establecido desde hace mucho tiempo: se sabe que los niveles elevados de PAl-1 están fuertemente correlacionados con la gravedad del síndrome. Los niveles de PAI-1 pueden reducirse con la restricción calórica, la pérdida de peso y la disminución de la grasa corporal ${ }^{44}$. Kraja, et al. mostraron una fuerte asociación entre el PAl-1 y los componentes del SM, incluyendo el IMC, los triglicéridos y la $\mathrm{Rl}^{47}$.

\section{Interleucina 6}

La IL-6 es una citocina proinflamatoria, forma parte de la respuesta inflamatoria natural y regularmente es secretada por los macrófagos $M 1^{48}$. Hay estudios que han descrito que durante la patogenia del SM frecuentemente se produce una disfunción de los adipocitos que conlleva el aumento de macrófagos M1 en el tejido adiposo. Esto puede resultar en una mayor secreción de IL-6 y otras citocinas proinflamatorias del tejido adiposo induciendo la $R I^{48}$. Además, la IL-6 se ha vinculado con la progresión de la disfunción cardíaca ${ }^{49}$. Como otros biomarcadores, la IL-6 se ha encontrado asociada con cada uno de los componentes del SM. En un estudio con pacientes obesos y no obesos con DM2 se encontró que los pacientes obesos con DM2 presentaban niveles más altos de IL-6 que los no obesos ${ }^{50}$. En otro estudio con mujeres posmenopáusicas se encontró una asociación de los niveles altos de IL-6 con la obesidad abdominal, niveles bajos de C-HDL y niveles altos de triglicéridos ${ }^{51}$. Esta asociación estrecha entre la IL-6, el SM y cada uno de sus componentes sugiere que es un factor importante durante la progresión del síndrome.

\section{Proteína $\mathbf{C}$ reactiva}

La PCR es una citocina producida por el hígado en su mayoría bajo el control transcripcional de la citocina IL-6. Habitualmente, cuando hay inflamación general en el cuerpo el nivel de PCR se encuentra aumentado52. La PCR es probablemente uno de los biomarcadores más estudiados hasta el momento. Numerosos estudios han confirmado que los niveles de PCR son elevados en pacientes con SM y pacientes en riesgo cardiovascular ${ }^{53,54}$. Además, se ha propuesto anexar la prueba de PCR de alta sensibilidad (PCRas) como criterio clínico para el $\mathrm{SM}^{54}$. En un estudio de Bennozzi, et al. con 467 sujetos se encontró que los individuos con SM presentaron niveles superiores de PCRas que aquéllos sin SM. Además se observó que la concentración de PCRas incrementaba a medida que aumentaba el número de componentes del SM ${ }^{55}$. La PCR posiblemente tenga un papel en la patogénesis del SM; por tal motivo, se debe tomar en consideración la incorporación de la prueba de PCR; esta medida podría resultar en la detección y reducción de pacientes con SM y de las complicaciones de éste.

\section{Interleucina 10}

La IL-10 es secretada por monocitos o macrófagos M256. Es una citocina antiinflamatoria, es decir, que juega un papel en la disminución y modulación de la inflamación sistémica ${ }^{57}$. Una de sus funciones es promover la remodelación de tejido normal después de una respuesta inflamatoria; además la IL-10 exhibe un efecto protector contra la aterogénesis ${ }^{48,58}$. Van Exel, et al. realizaron un estudio en adultos mayores en donde se encontró que los niveles bajos de IL-10 se asociaban con la RI y la DM2. Además, el estudio encontró que los niveles de IL-10 se encontraban inversamente relacionados con los niveles totales de colesterol, LDL, triglicéridos, glucosa en sangre y hemoglobina $\mathrm{A} 1 \mathrm{c}$, pero positivamente relacionados con los niveles de C-HDL ${ }^{59}$. La IL-10 es un componente clave del SM, ya que antagoniza las 
acciones proinflamatorias de la IL-6 y el TNF- $\alpha$. Esto sugiere que la IL-10 ejerce un efecto protector contra el aumento de estas citocinas. Sin embargo, algunos estudios han recomendado el uso combinado de diferentes citocinas, para de esta manera aumentar la sensibilidad y especificidad en la detección del SM.

\section{Factor de necrosis tumoral $\alpha$}

El TNF- $\alpha$ es una citocina proinflamatoria secretada por el tejido adiposo visceral; esto es una característica común en el SM ${ }^{60}$. Los niveles elevados de TNF- $\alpha$ se han asociado con la RI61. Por tal motivo, se ha sugerido que esta citocina puede participar tanto en el desarrollo como en la progresión del SM y las enfermedades asociadas al síndrome ${ }^{28,40}$. En un estudio realizado en adultos con SM se encontró una asociación entre los niveles elevados de TNF- $\alpha$ y otras citocinas proinflamatorias y la Rl y la hipertrigliceridemia. Los niveles de TNF- $\alpha$, IL-6 y leptina en estos pacientes fueron superiores a los del grupo de control, lo que indica que hay una correlación directa entre estas citocinas y el $\mathrm{SM}^{14}$. Balaçoiu, et al. sugieren que la detección temprana del estado inflamatorio (TNF- $\alpha$ e IL-6) de un individuo podría ser útil para el monitoreo y la intervención temprana del SM y sus comorbilidades ${ }^{62}$.

\section{Paraoxonasa}

La PON-1 es una enzima lipolactonasa asociada con el C-HDL capaz de prevenir la elevación de LDL y la oxidación de la membrana celular; por lo tanto, se considera que es una enzima ateroprotectora ${ }^{63}$. Se cree que las reducciones en la actividad del C-HDL y de la PON1 se encuentran en varias enfermedades con componentes inflamatorios; esta condición conduce a niveles disfuncionales de C-HDL, lo que conlleva inflamación y aterosclerosis ${ }^{64}$. Debido a sus propiedades antioxidantes, la PON-1 puede utilizarse como biomarcador para evaluar la capacidad antioxidante, la tendencia a la inflamación sistémica y la disfunción vascular. En un estudio con adolescentes con sobrepeso y obesidad se encontró que los niveles disminuidos de PON-1 se asociaban a obesidad central y SM. Además, los niveles más bajos de PON-1 se asociaron con hipertensión arterial, hipertrigliceridemia, RI, tolerancia a la glucosa alterada y aumento del estrés oxidativo ${ }^{63}$. La literatura sugiere que la PON-1 podría desempeñar un papel importante en la atenuación de los componentes del SM que surgen como resultado del estrés oxidativo.

\section{LDL oxidada}

La LDLox es un producto de la oxidación de lípidos y actualmente se ha demostrado que es un marcador del estrés oxidativo. Los niveles disfuncionales de LDLox resultan en daño celular $u$ apoptosis ${ }^{65}$. Se ha encontrado que la LDLox es clave en la iniciación y progresión de la aterogénesis ${ }^{6}$. Los estudios han demostrado que los niveles de LDLox son significativamente elevados en los pacientes con SM ${ }^{67}$. Además, los niveles elevados de LDLox se asocian con la reducción de la elasticidad arterial, que es un factor de riesgo para el desarrollo de enfermedad coronaria68. Un estudio en adolescentes latinos encontró una asociación de los niveles elevados de LDLox con el aumento de la adiposidad y la R/69. La literatura sugiere que la LDLox representa un biomarcador prometedor para la detección del SM y sus componentes durante el desarrollo y progresión del síndrome.

\section{DISCUSIÓN}

Este estudio ha reunido la literatura y evidencia científica actuales que describen la interrelación entre algunos de los biomarcadores conocidos hasta el momento y el SM. Nuestro país presenta uno de los índices más altos de obesidad y sobrepeso a nivel mundial, de acuerdo con un informe de la Organización de las Naciones Unidas para la Alimentación (FAO): México se halla en segundo lugar, con la mayor tasa de sobrepeso en América Latina y el Caribe, sólo superado por las Bahamas. Por lo tanto, la caracterización de un grupo de biomarcadores con validez clínica que puedan pronosticar y establecer los individuos en riesgo de padecer SM es urgente y necesaria, no sólo para el tratamiento, sino también para la disminución de las prevalencias del síndrome y de sus enfermedades asociadas.

El SM es una condición multifactorial, la cual frecuentemente resulta de la obesidad, pero el mecanismo exacto todavía está por determinarse. Una de las características distintivas de la obesidad es el estrés oxidativo que conlleva la inflamación crónica de bajo grado que resulta en la alteración del metabolismo de los lípidos con afección a la sensibilidad de la insulina (Fig. 1).

Se cree que la inducción del estrés oxidativo sistémico es en parte responsable de la secreción desregulada de adipocinas que contribuye al desarrollo del SM. Se 


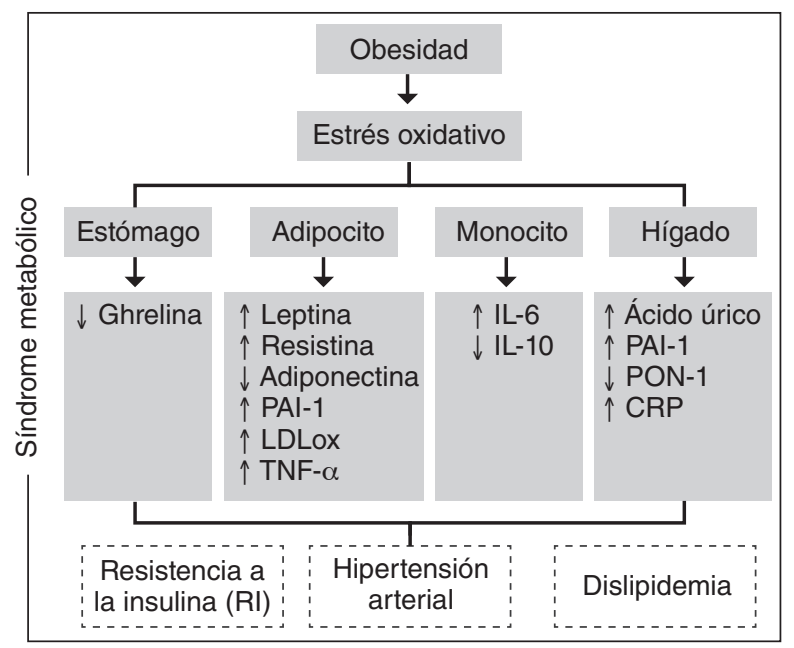

Figura 1. Representación esquemática de la interrelación de los biomarcadores en el SM.

ha demostrado que la leptina, la RSTN, el PAl-1, el ácido úrico, la IL-6, la PCR, el TNF- $\alpha$ y la LDLox se encuentran elevados en el SM y generalmente están correlacionados con un mayor número de componentes presentes en el SM. Por otra parte, los niveles de adiponectina, ghrelina, IL-10 y PON-1 se encuentran disminuidos en el SM (Tabla 2).

En un estudio realizado por Bae, et al. en 199 sujetos en donde se examinó la relación entre el incremento de adipocinas y citocinas inflamatorias y el riesgo de padecer SM se encontró una asociación significativa con el TNF- $\alpha$ (odds ratio [OR]: 4.78; $p<0.01$ ), la IL-6 (OR: 7.60; $\mathrm{p}<0.0001)$ y la PCR (OR: 2.72; $\mathrm{p}<0.05)^{70}$ (Tabla 3).

El uso potencial de múltiples biomarcadores para el diagnóstico, la detección temprana, la personalización del tratamiento e incluso la esquematización de los riesgos aún no ha sido explorado en su totalidad. A pesar de que existen muchos estudios sobre biomarcadores de manera individual, hay una laguna en la investigación sobre el resultado de la interacción de múltiples biomarcadores en el SM.

\section{CONCLUSIÓN}

Hasta el momento no se ha identificado un único mecanismo fisiopatológico relacionado con el SM o algunos de sus componentes; por lo tanto, es necesario realizar nuevos estudios que puedan esclarecer la
Tabla 2. Niveles y efectos de los biomarcadores en el SM

\begin{tabular}{|c|c|c|}
\hline Biomarcadores & SM & Efecto metabólico \\
\hline Leptina & $\uparrow$ & $\begin{array}{l}\uparrow \text { Gasto de energía } \\
\uparrow \text { Sensibilidad a la insulina } \\
\uparrow \text { Oxidación de ácidos grasos } \\
\downarrow \text { Apetito } \\
\downarrow \text { Acumulación de grasa }\end{array}$ \\
\hline RSTN & $\uparrow$ & $\downarrow$ Sensibilidad a la insulina \\
\hline Adiponectina & $\downarrow$ & $\begin{array}{l}\uparrow \text { Gasto de energía } \\
\uparrow \text { Sensibilidad a la insulina } \\
\uparrow \text { Oxidación de ácidos grasos }\end{array}$ \\
\hline Ghrelina & $\downarrow$ & $\begin{array}{l}\uparrow \text { Apetito } \\
\uparrow \text { Lipólisis }\end{array}$ \\
\hline Ácido úrico & $\uparrow$ & $\begin{array}{l}\uparrow \text { Oxidación de ácidos grasos } \\
\uparrow \text { Inflamación } \\
\uparrow \text { Triglicéridos }\end{array}$ \\
\hline PAl-1 & $\uparrow$ & $\uparrow$ Sensibilidad a la insulina \\
\hline IL-6 & $\uparrow$ & $\begin{array}{l}\uparrow \text { Gasto de energía } \\
\downarrow \text { Sensibilidad a la insulina } \\
\downarrow \text { Apetito }\end{array}$ \\
\hline PCR & $\uparrow$ & $\begin{array}{l}\uparrow \text { Inflamación } \\
\uparrow \text { Estrés oxidativo }\end{array}$ \\
\hline IL-10 & $\downarrow$ & $\begin{array}{l}\downarrow \text { Inflamación } \\
\downarrow \text { Triglicéridos }\end{array}$ \\
\hline TNF- $\alpha$ & $\uparrow$ & $\begin{array}{l}\downarrow \text { Sensibilidad a la insulina } \\
\downarrow \text { Apetito }\end{array}$ \\
\hline PON-1 & $\downarrow$ & $\begin{array}{l}\uparrow \text { Sensibilidad a la insulina } \\
\uparrow \text { Estrés oxidativo }\end{array}$ \\
\hline LDLox & $\uparrow$ & $\begin{array}{l}\uparrow \text { Sensibilidad a la insulina } \\
\uparrow \text { Dislipidemia } \\
\uparrow \text { Estrés oxidativo }\end{array}$ \\
\hline
\end{tabular}

Tabla 3. Odds ratio para el SM según los biomarcadores*

\begin{tabular}{lcr}
\hline & OR (IC 95\%) & Valor $\mathbf{p}$ \\
\hline Adiponectina & $0.42(0.18-0.94)$ & 0.0355 \\
RSTN & $1.60(0.71-3.63)$ & 0.2614 \\
Leptina & $1.49(0.63-3.52)$ & 0.3592 \\
LDLoX & $1.82(0.76-4.40)$ & 0.1843 \\
TNF- $\alpha$ & $4.78(1.90-12.28)$ & 0.0090 \\
IL-6 & $7.60(2.99-19.31)$ & $<0.0001$ \\
PCR & $2.72(1.15-6.42)$ & 0.0228 \\
\hline
\end{tabular}

*Las OR se ajustaron por edad, sexo, IMC y tabaquismo.

IC: intervalo de confianza.

Adaptado de Bae, et al..$^{70}$.

funcionalidad de diversos biomarcadores para el diagnóstico y tratamiento del SM. El SM representa una carga significativa para la salud pública, especialmente en México, debido a las altas prevalencias de obesidad, hipertensión y diabetes. Por ello, la identificación de un grupo de biomarcadores proporcionaría una herramienta de fácil acceso y mínima invasión para la detección y control de esta enfermedad en nuestro país. 
Con base en la evidencia presentada en este estudio, algunos biomarcadores mencionados se correlacionan significativamente con el SM y podrían proporcionar un medio mínimamente invasivo para la detección temprana y tratamiento específico del síndrome y los trastornos asociados a esta enfermedad. Sin embargo, se recomienda la realización de investigación adicional para determinar la eficacia de la aplicación de estos biomarcadores en el diagnóstico y tratamiento en un entorno clínico.

\section{BIBLIOGRAFÍA}

1. Srikanthan K, Feyh A, Visweshwar H, Shapiro JI, Sodhi K. Systematic review of metabolic syndrome biomarkers: a panel for early detection, management, and risk stratification in the West Virginian population. Int J Med Sci. 2016;13(1):25.

2. Acosta García E. Obesidad, tejido adiposo y resistencia a la insulina. Acta Bioquímica Clínica Latinoamericana. 2012;46(2):183-94.

3. Lizarzaburu Robles JC, ed. Síndrome metabólico: concepto y aplicación práctica. An Fac Med. Facultad de Medicina, UNMSM; 2013.

4. Truthmann J, Mensink GB, Bosy-Westphal A, Scheidt-Nave C, Schienkiewitz A. Metabolic Health in Relation to Body Size: Changes in Prevalence over Time between 1997-99 and 2008-11 in Germany. PloS One. 2016; 11(11):e0167159.

5. Grundy SM, Brewer Jr H, Cleeman JI, Smith Jr S, Lenfant C, for the Conference Participants. Definition of metabolic syndrome. Report of the National Heart, Lung, and Blood Institute/American Heart Association Conference on scientific issues related to definition. Circulation. 2004;109(3):433-8

6. Ehrmann DA, Liljenquist DR, Kasza K, Azziz R, Legro RS, Ghazzi MN, et al. Prevalence and predictors of the metabolic syndrome in women with polycystic ovary syndrome. J Clin Endocrinol Metab. 2006;91(1):48-53.

7. Esposito K, Chiodini P, Colao A, Lenzi A, Giugliano D. Metabolic syndrome and risk of cancer. Diabetes Care. 2012;35(11):2402-11.

8. González-Chávez A, Simental L, Elizondo-Argueta S, Sánchez J, Gutiérrez Salgado J, Guerrero-Romero F. Prevalencia del síndrome metabólico entre adultos mexicanos no diabéticos, usando las definiciones de la OMS, NCEPATPIIla e IDF. Rev Med Hosp Gen Mex. 2008;71(1):11-9.

9. México: INSP INdSP. Encuesta Nacional de Salud y Nutrición 2016. 2016.

10. Etzioni R, Urban N, Ramsey S, McIntosh M, Schwartz S, Reid B, et al. The case for early detection. Nat Rev Cancer. 2003:3(4):243.

11. López-Jaramillo P, Pradilla LP, Bracho Y. Papel del adipocito en la inflamación del síndrome metabólico. Acta Médica Colombiana. 2005; 30(3):137-40.

12. Deng $Y$, Scherer PE. Adipokines as novel biomarkers and regulators of the metabolic syndrome. Ann N Y Acad Sci. 2010;1212:E1-E19.

13. Furukawa S, Fujita T, Shimabukuro M, Iwaki M, Yamada $Y$, Nakajima $Y$, et al. Increased oxidative stress in obesity and its impact on metabolic syndrome. J Clin Invest. 2017;114(12):1752-61.

14. Pedersen M, Bruunsgaard $H$, Weis N, Hendel HW, Andreassen BU, Eldrup $\mathrm{E}$, et al. Circulating levels of TNF-alpha and IL-6-relation to truncal fat mass and muscle mass in healthy elderly individuals and in patients with type-2 diabetes. Mech Ageing Dev. 2003;124(4):495-502.

15. Dong M, Ren J. What fans the fire: insights into mechanisms of leptin in metabolic syndrome-associated heart diseases. Curr Pharm Des. 2014; 20(4):652-8.

16. De Luis D, Perez CJ, Duenas A. Leptin and obesity. Minerva Med. 2009:100(3):229-36.

17. Thøgersen AM, Söderberg S, Jansson JH, Dahlén G, Boman K, Nilsson TK, et al. Interactions between fibrinolysis, lipoproteins and leptin related to a first myocardial infarction. Eur J Cardiovasc Prev Rehabil. 2004;11(1):33-40.

18. Ghantous C, Azrak Z, Hanache S, Abou-Kheir W, Zeidan A. Differential role of leptin and adiponectin in cardiovascular system. Int J Endocrinol. 2015; 2015.

19. García- Jiménez S, Bernal Fernández G, Martínez Salazar MF, Monroy Noyola A, Toledano Jaimes C, Meneses Acosta A, et al. Serum leptin is associated with metabolic syndrome in obese Mexican subjects. J Clin Lab Anal. 2015; 29(1):5-9.

20. Nogueiras R, González CR, Mendieta H, Lage R, Diéguez C. Resistina: una nueva hormona expresada en el tejido adiposo. Rev Esp Obes. 2005; 3(4):194-211.

21. Steppan CM, Bailey ST, Bhat S, Brown EJ. The hormone resistin links obesity to diabetes. Nature. 2001;409(6818):307.
22. Pfützner $A$, Langenfeld $M$, Kunt $T$, Löbig $M$, Forst $T$. Evaluation of human resistin assays with serum from patients with type 2 diabetes and different degrees of insulin resistance. Clin Lab. 2003;49(11-12):571-6.

23. Grundy SM. Obesity, metabolic syndrome, and cardiovascular disease. J Clin Endocrinol Metab. 2004;89(6):2595-600.

24. Steppan CM, Lazar MA. The current biology of resistin. J Int Med. 2004; 255(4):439-47

25. Ziemke F, Mantzoros CS. Adiponectin in insulin resistance: lessons from translational research. Am J Clin Nutr. 2010;91(1):258S-61S.

26. Lara-Castro C, Fu Y, Chung BH, Garvey WT. Adiponectin and the metabolic syndrome: mechanisms mediating risk for metabolic and cardiovascular disease. Curr Opin Lipidol. 2007:18(3):263-70.

27. Karbowska J, Kochan Z. Role of adiponectin in the regulation of carbohydrate and lipid metabolism. J Physiol Pharmacol. 2006;57:103.

28. Palomer $X$, Pérez A, Blanco-Vaca $F$. Adiponectina: un nuevo nexo entre obesidad, resistencia a la insulina y enfermedad cardiovascular. Med Clin (Barc). 2005;124(10):388-95

29. Hui X, Lam KS, Vanhoutte PM, Xu A. Adiponectin and cardiovascular health: an update. Br J Pharmacol. 2012;165(3):574-90.

30. Kawano J, Arora R. The role of adiponectin in obesity, diabetes, and cardiovascular disease. J Cardiometab Syndr. 2009;4(1):44-9.

31. Chaila M, Fabio S, Quiroga E, Sánchez de Boeck N, Namur M, D'urso M, et al. Prevalencia de síndrome metabólico en niños y adolescentes según diferentes criterios diagnósticos y su correlación con niveles de leptina y adiponectina. Rev Argent Endocrinol Metab. 2012;49(3):103-14.

32. Varela L, Vázquez MJ, Cordido F, Nogueiras R, Vidal-Puig A, Diéguez C, et al. Ghrelin and lipid metabolism: key partners in energy balance. J Mol Endocrinol. 2011;46(2):R43-R63.

33. Kishimoto I, Tokudome T, Hosoda H, Miyazato M, Kangawa K. Ghrelin and cardiovascular diseases. J Cardiol. 2012:59(1):8-13.

34. Tesauro M, Schinzari F, Rovella V, Di Daniele N, Lauro D, Mores N, et al. Ghrelin restores the endothelin 1/nitric oxide balance in patients with obesity-related metabolic syndrome. Hypertension. 2009;54(5):995-1000.

35. Pulkkinen L, Ukkola 0, Kolehmainen M, Uusitupa M. Ghrelin in diabetes and metabolic syndrome. Int J Pept. 2010;2010.

36. Ukkola 0. Ghrelin and the metabolic balance. J Endocrinol Invest. 2005;28(11):849-52.

37. Tschöp M, Weyer C, Tataranni PA, Devanarayan V, Ravussin E, Heiman ML. Circulating ghrelin levels are decreased in human obesity. Diabetes. 2001:50(4):707-9.

38. Fagerberg B, Hultén LM, Hulthe J. Plasma ghrelin, body fat, insulin resistance, and smoking in clinically healthy men: the atherosclerosis and insulin resistance study. Metabolism. 2003;52(11):1460-3.

39. Billiet L, Doaty S, Katz JD, Velasquez MT. Review of hyperuricemia as new marker for metabolic syndrome. ISRN Rheumatol. 2014;2014.

40. de Candia AM, Júnior HV, Mesquita ET. Ativação imune-inflamatória na insuficiência cardíaca. Arq Bras Cardiol. 2007;89(3):201-8.

41. Martínez A, González A, Cerda C, Pérez P, Castro P, Pérez 0, et al. [Prognostic value of hyperuricemia in chronic heart failure]. Rev Med Chil. 2004; 132(9):1031-6

42. Silva HAd, Carraro JCC, Bressan J, Hermsdorff HHM. Relation between uric acid and metabolic syndrome in subjects with cardiometabolic risk. Einstein (Sao Paulo). 2015;13(2):202-8

43. Jiménez AER, Navarro J, Islas MC, DFranchis LJC, Gómez JL, Pichardo CN, et al. Prevalencia del síndrome metabólico en relación con las concentraciones de ácido úrico. Medicina Interna de México. 2009;25(4):278-84.

44. Binder BR, Christ G, Gruber F, Grubic N, Hufnagl P, Krebs M, et al. Plasminogen activator inhibitor 1: physiological and pathophysiological roles. Physiology. 2002:17(2):56-61.

45. Ciszek A, Bermúdez V, Leal E, Bermúdez F, Cano R, Finol F, et al. Inhibidor del activador del plasminógeno tipo 1 (PAl-1) y su relación con la aterosclerosis coronaria. Revista Latinoamericana de Hipertensión. 2007;2(5).

46. Juhan- Vague I, Alessi MC, Mavri A, Morange P. Plasminogen activator inhibitor- 1 , inflammation, obesity, insulin resistance and vascular risk. J Thromb Haemost. 2003;1(7):1575-9.

47. Kraja AT, Province MA, Arnett D, Wagenknecht L, Tang W, Hopkins PN, et al. Do inflammation and procoagulation biomarkers contribute to the metabolic syndrome cluster? Nutr Metab (Lond). 2007;4(1):28.

48. Aroor AR, McKarns S, DeMarco VG, Jia G, Sowers JR. Maladaptive immune and inflammatory pathways lead to cardiovascular insulin resistance. Metabolism. 2013;62(11):1543-52.

49. Harmon ME, Campen MJ, Miller C, Shuey C, Cajero M, Lucas S, et al. Associations of circulating oxidized LDL and conventional biomarkers of cardiovascular disease in a cross-sectional study of the Navajo population. PloS one. 2016;11(3):e0143102.

50. Goyal R, Faizy AF, Siddiqui SS, Singhai M. Evaluation of TNF- $\alpha$ and IL-6 levels in obese and non-obese diabetics: pre-and postinsulin effects. N Am J Med Sci. 2012;4(4):180-4

51. Fenkci S, Rota S, Sabir N, Sermez Y, Guclu A, Akdag B. Relationship of serum interleukin- 6 and tumor necrosis factor $\alpha$ levels with abdominal fat distribution evaluated by ultrasonography in overweight or obese postmenopausal women. J Investig Med. 2006;54(8):455-60 
52. Volanakis JE. Human C-reactive protein: expression, structure, and function. Mol Immunol. 2001;38(2):189-97.

53. Collaboration ERF. C-reactive protein concentration and risk of coronary heart disease, stroke, and mortality: an individual participant meta-analysis. Lancet. 2010;375(9709):132-40.

54. Sattar N, Gaw A, Scherbakova 0, Ford I, O'Reilly DSJ, Haffner SM, et al. Metabolic syndrome with and without $\mathrm{C}$-reactive protein as a predictor of coronary heart disease and diabetes in the West of Scotland Coronary Prevention Study. Circulation. 2003;108(4):414-9.

55. Benozzi SF, Perruzza F, Pennacchiotti GL. C-Reactive Protein: A Biomarker Associated with the Metabolic Syndrome and Abdominal. Argentine Journal of Cardiology. 2012;80(6):446-51

56. Xu H. Obesity and metabolic inflammation. Drug Discov Today Dis Mech. 2013;10(1):e21-e5.

57. Tsatsanis C, Zacharioudaki V, Androulidaki A, Dermitzaki E, Charalampopoulos I, Minas V, et al. Adiponectin induces TNF- $\alpha$ and IL-6 in macrophages and promotes tolerance to itself and other pro-inflammatory stimuli. Biochem Biophys Res Commun. 2005;335(4):1254-63.

58. Nishida M, Moriyama T, Sugita Y, Yamauchi-Takihara K. Interleukin-10 associates with adiponectin predominantly in subjects with metabolic syndrome. Circ J. 2007:71(8):1234-8.

59. van Exel E, Gussekloo J, de Craen AJ, Frölich M, Bootsma-van der Wiel A, Westendorp RG. Low production capacity of interleukin-10 associates with the metabolic syndrome and type 2 diabetes. Diabetes. 2002;51(4): 1088-92.

60. Musialik K. The influence of chosen adipocytokines on blood pressure values in patients with metabolic syndrome. Kardiol Pol. 2012;70(12):1237-42.

61. Ritchie S, Connell J. The link between abdominal obesity, metabolic syndrome and cardiovascular disease. Nutr Metab Cardiovasc Dis. 2007;17(4):319-26.
62. Balaçoiu M, Balaçoiu AT, Stepan AE, Dinescu SN, Avramescu CS, Dumitrescu $\mathrm{D}$, et al. Proatherogenic adipocytokines levels in metabolic syndrome. Romanian J Morphol Embryol Rev Roum Morphol Embryol. 2014;55(1):29-33.

63. Krzystek-Korpacka M, Patryn E, Hotowy K, Czapinska E, Majda J, Kustrzeba W. Paraoxonase-1 activity in overweight and obese children and adolescents: association with obesity-related inflammation and oxidative stress. Adv Clin Exp Med. 2013;22(2):229-36.

64. Mackness M, Mackness B. Human paraoxonase-1 (PON1): Gene structure and expression, promiscuous activities and multiple physiological roles. Gene. 2015;567(1):12-21.

65. Landar A, Zmijewski JW, Dickinson DA, Le Goffe C, Johnson MS, Milne GL, et al. Interaction of electrophilic lipid oxidation products with mitochondria in endothelial cells and formation of reactive oxygen species. Am J Physiol Heart Circ Physiol. 2006;290(5):H1777-H87.

66. Carvajal Carvajal C. LDL oxidada y la aterosclerosis. Medicina Legal de Costa Rica. 2015;32(1):161-9.

67. Osman MT, Rahman T, Ismail T, Azlina A, Nawawi H. Investigation of Oxidative Stress Status in Metabolic Syndrome Patients Using Lipid Peroxidation Biomarkers. International Archives of Medicine. 2016;9.

68. Pohjantähti-Maaroos H, Palomäki A, Kankkunen P, Laitinen R, Husgafvel S, Oksanen K. Circulating oxidized low-density lipoproteins and arterial elasticity: comparison between men with metabolic syndrome and physically active counterparts. Cardiovasc Diabetol. 2010;9(1):41.

69. Ryder JR, Vega-López S, Djedjos CS, Shaibi GQ. Abdominal adiposity, insulin resistance, and oxidized low-density lipoproteins in Latino adolescents. Diabetol Metab Syndr. 2013;5(1):72.

70. Bae YJ, Kim SH, Chung JH, Song SW, Kim KS, Kim MK, et al. Evaluation of adiposity-related biomarkers as metabolic syndrome indicators. Clin Nutr Res. 2013;2(2):91-9. 